\title{
Better fed wasps are more selfish
}

\section{H. Richards ${ }^{1}$}

Published online: 29 October 2021

(c) International Union for the Study of Social Insects (IUSSI) 2021
Links between nutritional and reproductive status are well established in social insects. Best known are effects of larval nutrition on caste development in highly eusocial insects. There is also considerable evidence in primitively social insects that larval diets influence developmental trajectories culminating in differentiated queens and workers. However, in many primitively eusocial insects, caste identity is not really solidified until adulthood, sometimes days or weeks after eclosion. Even then, behavioural flexibility among both queens and workers implies that a variety of environmental and social factors influence the final stages of caste development. Factors associated with caste determination in primitively social wasps and bees include seasonal timing (especially in temperate zone species), the social environment of the nest into which a newly eclosed female emerges, and food availability. Often these processes reinforce the developmental trajectories previously induced by larval experience, but sometimes the opposite may happen, with larval trajectories reversed during the adult stages.

The dominance-nutrition hypothesis (Markiewicz and O'Donnell 2001) suggests how adult nutrition may influence caste and dominance behaviour of primitively eusocial wasps. The dominance-nutrition hypothesis originally proposed a feedback model causally linking dominance status, task performance, nutritional status, and ovary development (Markiewicz and O'Donnell 2001). In primitively eusocial wasp colonies, food foragers bring insect prey back to the nest, delivering it both to non-foraging adult nestmates and to developing juveniles (larvae). Foraging is a risky and energetically expensive task, which in primitively social wasp colonies, tends to be performed by more subordinate workers, whereas more dominant workers can remain on the nest and take food from returning foragers. By avoiding the energetic costs of foraging, dominant workers may be

M. H. Richards

mrichards@brocku.ca

1 Brock University, St. Catharines, Canada better able to sequester the physiological resources for egg production.

In this issue, Jithu Krishnan, Anindita Brahma, Sayali Chavan, and Raghavendra Gadagkar (Krishnan et al. 2021) present the first experimental test of the dominance-nutrition hypothesis, focussing on Ropalidia marginata, a species seemingly happy to live in large, walk-in cages that enable intensive behavioural observations of marked individuals. Captive wasps were provided with an ad libitum supply of rice moth larvae, dilute honey and water. In control colonies, colonies were dependent on foraging workers to bring food back to the nest, but in experimental colonies, the biologists themselves supplemented the available food by hand-feeding each individually marked adult twice a day with as many rice moth larvae as it would accept. Thus, in experimental colonies, all wasps could consume large amounts of food without having either to forage or to take it from other foragers. Apparently, it is as hard for wasps to resist free food as it is for humans - the experimental wasps consumed almost twice as much food as did control wasps. Not only did they eat more, but experimental wasps behaved differently. Queen turnover was more frequent in experimental colonies and more new nests were initiated by females that left their natal colony. Females from the better fed experimental colonies were less likely to remain as helpers on the natal nest, and more likely to become reproductives.

Ultimately, in $R$. marginata, being released from nutritional constraints allowed more females to behave selfishly, rather than cooperatively. Nutritional status connects the behaviour of females in colonies to their local environment, in which food availability can vary enormously from season to season or even over the lifespan of a colony. It would be interesting to investigate under field conditions, whether wasps with more access to food are more likely to behave selfishly. Such a link between food and selfish behaviour has been proposed for primitively eusocial sweat bees. When pollen is abundant, sweat bee workers can provision brood cells faster than queens can lay eggs. Under these circumstances, workers are more likely to act as selfish reproductives, even in the presence of a viable queen (Schwarz et al. 
2007). Perhaps further research will demonstrate that the dominance-nutrition hypothesis can help to explain the frequencies of selfish and helpful workers across primitively eusocial insects.

\section{References}

Krishnan JU, Brahma A, Chavan SK, Gadagkar R (2021) Nutrition induced direct fitness for workers in a primitively eusocial wasp. Insect Soc. https://doi.org/10.1007/s00040-021-00835-3
Markiewicz D, O’Donnell S (2001) Social dominance, task performance and nutrition: implications for reproduction in eusocial wasps. J Comp Physiol A 187:327-333. https://doi.org/10.1007/ s003590100204

Schwarz MP, Richards MH, Danforth BN (2007) Changing paradigms in insect social evolution: insights from halictine and allodapine bees. Annu Rev Entomol 52:127-150. https://doi.org/10.1146/ annurev.ento.51.110104.150950 